\title{
Inherent Fabrication Yields and Asymmetries Impacts on MZI- SOA Static Modelling
}

\author{
Giorgia Parca $^{+\#}$, Rogério Dionísio*\#, Member, IEEE, Cláudia Reis ${ }^{\#}$, Silvello Betti ${ }^{+}$, Giorgio Tosi Beleffi ${ }^{1}$, \\ António Teixeira", Member, IEEE \\ "Instituto de Telecomunicações, Campus de Santiago, 3810-197, Aveiro, Portugal \\ + Università di Roma "Tor Vergata” Dipartimento di Ingegneria Elettronica, Rome, Italy \\ ${ }^{1}$ ISCOM Istituto Superiore delle Comunicazioni e Tecnologie dell'Informazione, Rome, Italy \\ * Escola Superior de Tecnologia, Instituto Politécnico de Castelo Branco, 6000-767 Castelo Branco, Portugal \\ Tel.003906 72597447,Fax: 003906 72597435,E-mail: parca@ing.uniroma2.it
}

\begin{abstract}
The characteristics of MZI-SOA are affected by its internal design yields and asymmetries, leading to an unbalanced power distribution at the output arms and difficult or non optimum biasing. Therefore, we propose an experimental methodology and model for MZI-SOA structures taking into account internal asymmetries as well as the internal SOA behaviour under different current both as what regards amplitude and phase.
\end{abstract}

Keywords: SOA, Mach-Zehnder SOA interferometer, splitting factors, extinction ratio, all optical processing

\section{INTRODUCTION}

Integrated MZI-SOAs (Mach-Zehnder Semiconductor Optical amplifiers) [1] are compact devices with a vast potential for application in several optical domains. A schematic of the internal structure of a MZI-SOA is depicted in Fig. 1 (inset). They can be used in logical gates [2], digital phase modulation [3], switching and wavelength conversion [4], all-optical processing and signal regeneration [5], among others.

In all these applications, the interferometer must be previously biased to set an operational state in terms of output power and power/phase change as a function of the available inputs, and depending on the application in hands. However most of these biasing points require very long and difficult initial setup phase, which varies from device to device, due to its internal fabrication yields. The MZI-SOA is an integrated device (the used ones are hybridly integrated, resulting in increased yields but more flexibility), which encompasses several types of couplers, guides and SOA's (see Fig. 1 inset) each with its own tolerances and asymmetries (e.g. the couplers are not exactly $50 \%$ splitting ratio). These issues have some implications in many MZI-SOA functionalities, for example on the maximization of extinction ratio (ER) between the interferometer output ports.

This paper presents a black box model based on a set of functions and a methodology for evaluating the MZISOA relevant factors just based on external power measurements and $\mathrm{CW}$ laser. This model will allow extracting the coupling factors and relevant gain functions which contribute for the determination of the MZISOA behaviour, knowing that most of these parameters are hidden since they are masked in the output power which are normally a sum or difference of internal fields which are changed in amplitude and phase.

This paper is organized as follows. Section 2 shows the experimental setup used for all the required measurements and describes the MZI-SOA operational parameters. In Section 3 the couplers are characterized in terms of their real splitting factors. Section 4 presents the models behind the output power and their validation as a function of SOAs biasing currents.

\section{EXPERIMENTAL SETUP}

Due to the interferometric structure of the device, the power distribution along the device will affect the output interference depth and its potential optimization. Therefore, it is critical to first have a passive parts characterization. In order to carry out the analysis on MZI-SOA asymmetry properties, we used a 6 ports hybrid device, as shown in Fig. 1 inset. Each arm of the MZI incorporates one SOA and one Phase Shifter (PS). In order to assess each input-output path, two sets of switches are used, as seen in Fig. 1. Two sets of measurements were performed. The gain of one of the arms with the other arm blocked by a switched off SOA, and the SOA gain dependence on the biasing current. A DFB laser @1546.12 nm is used as the input signal. All power measurements were obtained through a Power Meter (PM).

\section{EXPERIMENTAL COUPLERS CHARACTERIZATION}

For all measurements, PS1 and PS2 are switched off, this has little impact, only may result on a phase unbalance, due to eventual difference in size or placement, when operating with the two SOA's biased. Also, in the optical side, the $\mathrm{CW}$ laser input power at the switches is equal to $3 \mathrm{dBm}$. When simply biased the SOAs current was by default $200 \mathrm{~mA}$. The measurements performed are summarized in Table 1. 


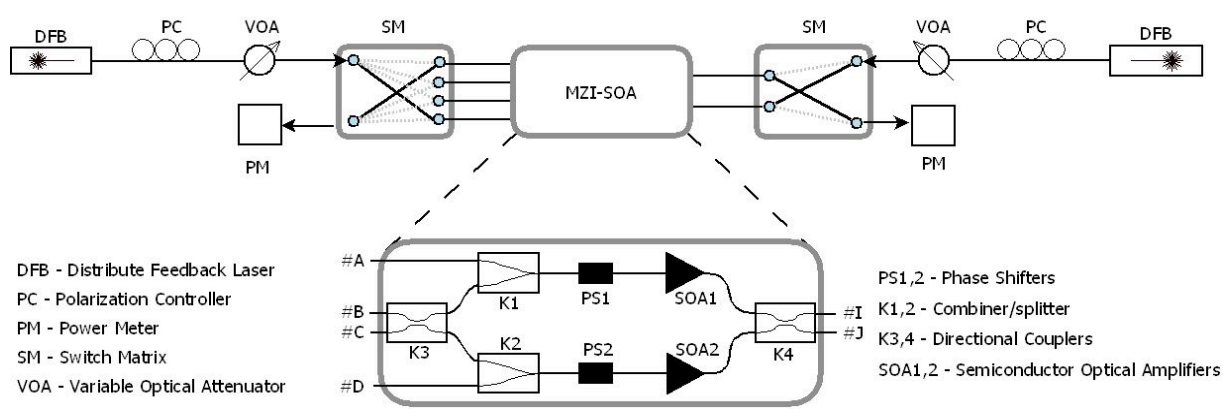

Figure 1. Experimental setup used to characterize the MZI-SOA with power measurements.

Table 1. Procedures for measurements and calculations.

\begin{tabular}{|c|c|c|c|c|c|c|}
\hline Coupler & CW port & PM port & I SOA1 & I SOA2 & Formulation & Splitting factor \\
\hline $\mathrm{K} 1$ & $\# \mathrm{I}$ & $\# \mathrm{~A}, \# \mathrm{~B}, \# \mathrm{C}$ & $200 \mathrm{~mA}$ & $0 \mathrm{~mA}$ & $\alpha_{1}=P_{\# A} /\left(P_{\# A}+P_{\# B}+P_{\# C}\right)$ & $52 \%$ \\
\hline $\mathrm{K} 2$ & $\# \mathrm{~J}$ & $\# \mathrm{~B}, \# \mathrm{C}, \mathrm{\# D}$ & $0 \mathrm{~mA}$ & $200 \mathrm{~mA}$ & $\alpha_{2}=P_{\# D} /\left(P_{\# B}+P_{\# C}+P_{\# D}\right)$ & $42 \%$ \\
\hline $\mathrm{K} 3$ & $\# \mathrm{I}$ & $\# \mathrm{~B}, \# \mathrm{C}$ & $200 \mathrm{~mA}$ & $0 \mathrm{~mA}$ & $\alpha_{3}=P_{\# B} /\left(P_{\# B}+P_{\# C}\right)$ & $52 \%$ \\
\hline $\mathrm{K} 4$ & $\# \mathrm{~A}$ & $\# \mathrm{I}, \# \mathrm{~J}$ & $200 \mathrm{~mA}$ & $0 \mathrm{~mA}$ & $\alpha_{4}=P_{\# I} /\left(P_{\# I}+P_{\# J}\right)$ & $60 \%$ \\
\hline
\end{tabular}

We can observe that couplers are not $50 \%-50 \%$ as ideally would be expected in an interferometric structure and their splitting factors are all different. This induces an asymmetric power distribution along the interferometer arms which in any operational point will cause second order effects like the different saturation of the SOA's in each of the arms.

Having all the passive paths characterized, it is now critical to have the active devices characterized. The phase shifters were considered linear, or at least able to be linearized, therefore not considered at all in the calculations. So, the next step of our analysis was the evaluation of MZI-SOA output power as function of input power and SOA current. These measurements were made, again considering the two arm separately, using the forward propagation, from input \#A to output \#I, with SOA2 disabled, and, similarly, from input \#B to output $\#$ J, with SOA1 disabled. The trends of the SOA output power vs. SOA current and input power are found and obtained curves are shown in Fig. 2 a) and Fig. 2 b). It is worth noting that this characterization is referred to the overall path on which the signal propagates, so it takes into account losses and any other kind of asymmetry of the device.

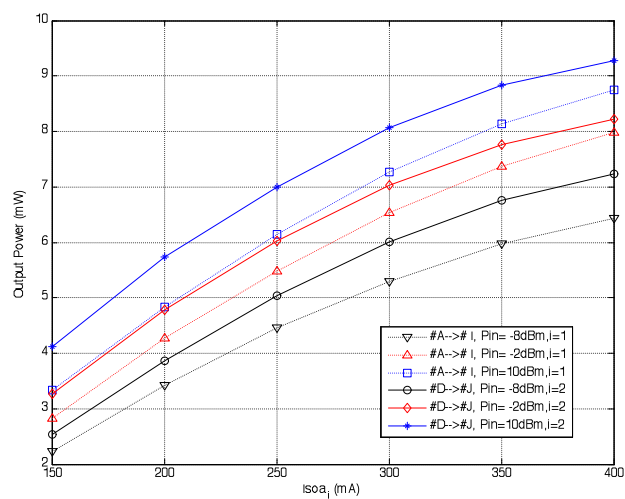

(a)

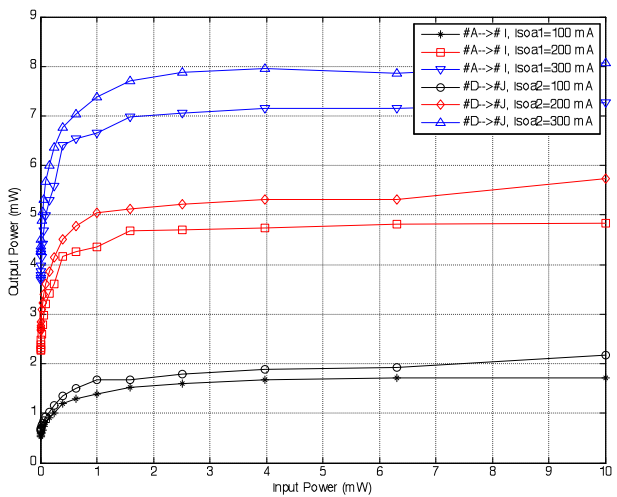

(b)

Figure 2. \#I, \#J Output power as function of (a) SOA1, SOA2 current variation and (b) \#A, \#D input power

\section{OUTPUT INTERFERENCE ANALYSIS}

The power at the output of the MZI-SOA's (\#I and \#J) is a result of an interference process occurring in coupler K4. The electromagnetic fields at this coupler two inputs will define the conditions for the outputs measured. So, specifically, when used as an amplitude modulator, one important factor to take into consideration is the Extinction Ration (ER) of the output signal. For example, through SOAs current variation, the power distribution on the MZI-SOA arms can be further changed, leading to the consequent variation of the interference conditions on output couplers.

Output power levels are highly dependent on SOA currents. As known and reported in the previous experimental analysis, SOAs gain is affected by current variation. Moreover, a phase shift on SOAs output signals is induced due to the dependence of SOAs refractive index on carrier density. 
Our aim is to characterize these effects and model the working characteristic for a MZI-SOA, in order to evaluate which parameters actually control the interference depth, thus providing a tool for operational point optimization, which for example can be used for maximizing ER.

Therefore, we present a relation between \#I and \#J output power as function of SOA1 current, obtained starting from interferometric structure principles considering the couplers yield and also some path difference:

$$
\begin{aligned}
& P_{I}\left(I_{\text {soa }}\right)=\left(1-\alpha_{4}\right) P_{1}\left(I_{\text {soo }}\right)+\alpha_{4} P_{2}\left(\text { Isoa }_{2}\right)-\sqrt{\alpha_{4}\left(1-\alpha_{4}\right) P_{1}\left(\text { Isoa }_{1}\right) P_{2}\left(\text { Isoa }_{2}\right)} \sin \left[\Delta \phi\left(\text { Isoa }_{1}\right)+\delta_{1}\right] \\
& P_{J}\left(I_{s o a_{1}}\right)=\left(1-\alpha_{4}\right) P_{2}\left(\text { Isoa }_{2}\right)+\alpha_{4} P_{1}\left(\text { Isoa }_{1}\right)+\sqrt{\alpha_{4}\left(1-\alpha_{4}\right) P_{1}\left(\text { Isoa }_{1}\right) P_{2}\left(\text { Isoa }_{2}\right)} \sin \left[\Delta \phi\left(I \operatorname{Isoa}_{1}\right)-\delta_{2}\right]
\end{aligned}
$$

Where

$$
\begin{aligned}
& P_{i}\left(\operatorname{Isoa}_{i}\right)=g_{i}\left(p_{i} \cdot \operatorname{Isoa}_{i}+q_{i}\right) \\
& \Delta \phi\left(\operatorname{Isoa}_{1}\right)=m \cdot I_{\text {soa }} \quad i=1,2
\end{aligned}
$$

$\alpha_{4}$ is the splitting factor of coupler K4.

$\mathrm{P} 1$ and $\mathrm{P} 2$ are power levels at the output of SOA1 and SOA2, respectively, and are dependent on current, which, for sake of simplicity and due to the range of currents used in the measurements, can be approximated through a linear curve, eq. (3), derived from experimental data (Figure 2). In particular, $g_{i}$ coefficient takes into account an adjustment of $\mathrm{P}_{\mathrm{i}}$, due to the experimental measurements made. $\Delta \phi$ is the phase shift induced by changing SOA1 current, again modelled through a linear approximation, eq. (4), because of the linear relation between SOA induced phase and carrier density, through refractive index variation. The $\delta_{i}$ parameter represent a phase mismatch on paths or coupler crossing factors, it affects the output interference by means of a reduction of maximum ER achievable.

To fit the remaining unknown factors, extra measurements were performed. A CW signal was coupled into input port \#B. We observe the dynamic of both the constructive and destructive interference respectively at the output port $\# \mathrm{I}$ and $\# \mathrm{~J}$, changing the current of SOA1, and fixing the SOA2 current at a reference value of $200 \mathrm{~mA}$

Fig. 3 shows experimental data compared with values estimated through eq. (1 and 2). In both cases we observe a misalignment between \#I and \#J minimum and maximum power levels. This is a clear result of the gain change in the SOA with the changing current and also to both the unbalanced splitting factors and phase shift of the couplers.

Moreover, the minimum of the \#I output power does not reach the zero, impacting the maximum achievable ER.

Table 2. Parameters extracted from the fittings and used in the final validation. Input power of $3 d B m$, fixed SOA2 current of $200 \mathrm{~mA}$.

\begin{tabular}{|c|c|c|c|c|c|c|c|c|}
\hline $\begin{array}{c}g_{1} \\
(-)\end{array}$ & $\begin{array}{c}g_{2} \\
(-)\end{array}$ & $\begin{array}{c}p_{1} \\
\left(\mathrm{~W} \cdot \mathrm{A}^{-1}\right)\end{array}$ & $\begin{array}{c}p_{2} \\
\left(\mathrm{~W}_{\mathrm{A}}^{-1}\right)\end{array}$ & $\begin{array}{c}q_{1} \\
(\mathrm{~W})\end{array}$ & $\begin{array}{c}q_{2} \\
(\mathrm{~W})\end{array}$ & $\begin{array}{c}m \\
\left(\mathrm{rad} . \mathrm{A}^{-1}\right)\end{array}$ & $\begin{array}{c}\delta_{1} \\
(\mathrm{rad})\end{array}$ & $\begin{array}{c}\delta_{2} \\
(\mathrm{rad})\end{array}$ \\
\hline 1.63 & 4.01 & $2.20 \cdot 10^{-2}$ & $2.33 \cdot 10^{-2}$ & $-5.01 \cdot 10^{-4}$ & $-3.56 \cdot 10^{-4}$ & 7.48 & $4.49 \cdot 10^{-2}$ & $1.57 \cdot 10^{-1}$ \\
\hline
\end{tabular}

From the analysis of Fig. 3 it is clear that the model prescribed, even considering the approximations made, eg, linear gain dependence of the SOA with the current, gives out a very precise fitting of the output power values. This is very important, since, from here one can capture the reasoning behind the major performance limitations of these structures, and based on this optimization of the behaviour having in mind the application target can be made. As Example, it can be said that the best operational point, without phase shifters adjustment, as an IM all-optical modulator, would most probably be achieved if the SOA1 would be biased at $250 \mathrm{~mA}$ 


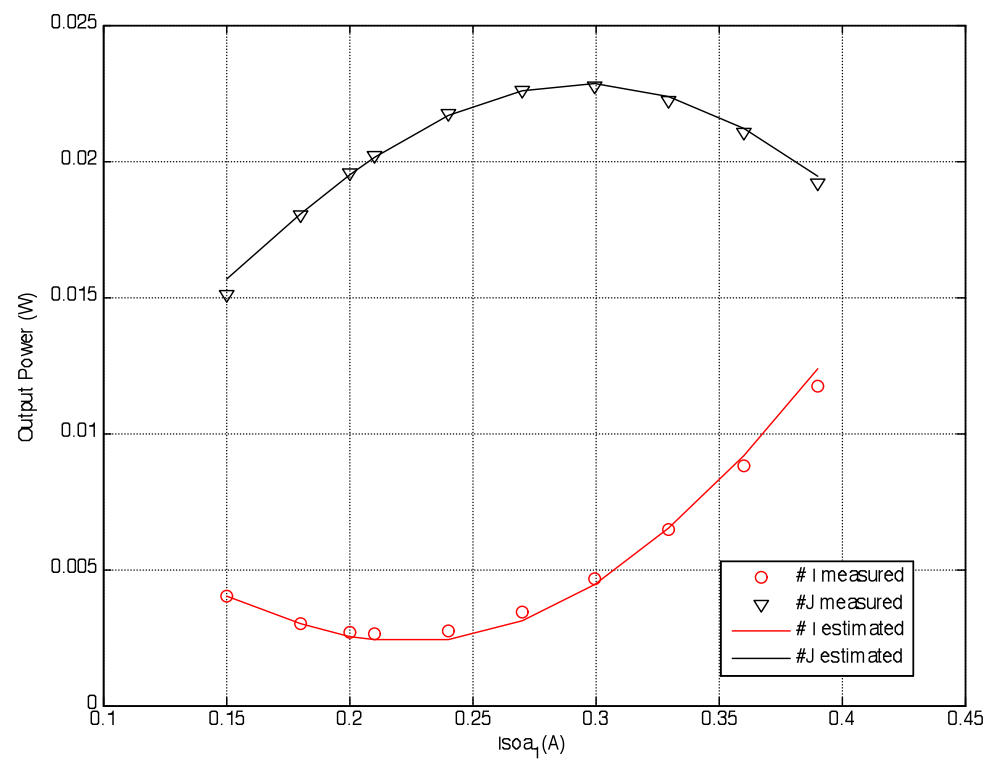

Figure 3. Measured and estimated power levels on \#I, \#J output ports with 3dBm optical input power at \#B.

\section{CONCLUSION}

The complete active and passive components of a MZI-SOA were characterized in static operational conditions. A model, which accurately predicts the output power as a function of the SOA current, has been produced and the methodology for extracting from output powers only all the required parameters have been given and presented. It is concluded that the yields in fabrication of these devices are quite high therefore strong variation of the coupling factors against the predicted optimum values should be expected and taken into consideration. Results obtained using proposed model agree with experimental data. The formulation allows to predict the achievable ER and to adjust operational conditions in order to optimize the MZI-SOA balancing.

\section{ACKNOWLEDGEMENTS}

The authors greatly acknowledge BONE and EURO-FOS networks of excellence and the PANORAMA Portuguese QREN project.

\section{REFERENCES}

[1] Maxwell, G., Poustie, A., Ford, C., Harlow, M., Townley, P., Nield, M., et al. (2005). Hybrid integration of monolithic semiconductor optical amplifier arrays using passive assembly. 55th Electronic Components \& Technology Conference, Vols 1 and 2, 2005 Proceedings, 1349-1352.

[2] Reis, C., Dionisio, R. P., Neto, B., Teixeira, A., \& Andre, P., "All-optical XOR based on integrated MZI-SOA with co- and counter-propagation scheme," in ICTON Mediterranean Winter Conference, 2009. ICTON-MW 2009. 3rd, 2009, pp. 1-4.

[3] Dionisio, R. P., Reis, C., Andre, P., Nogueira, R., \& Teixeira, A., "Experimental Study of a Phase Modulator Using an Active Interferometric Device," in IEEE Mediterranean Electrotechnical Conference, 2010. MELECON 2010. 15th, 2010.

[4] N. Yan, et al., "Simulation and Experimental Characterization of SOA-MZI-Based Multiwavelength Conversion," J. Lightwave Technol., vol. 27, pp. 117-127, 2009.

[5] Y. Xingwen, et al., "A Theoretical and Experimental Study on Modulation-Format-Independent Wavelength Conversion," J. Lightwave Technol., vol. 28, pp. 587-595, 2010. 